doi:10.17659/01.2019.0026

Journal of Case Reports 2019;9(2):103-105

\title{
Massive Hydrothorax Following External Jugular Vein Cannulation in an Infant
}

\author{
Stefania Franzini ${ }^{1}$, Emanuela Paradiso ${ }^{1}$, Claudia Casiraghi ${ }^{1}$, Stefano Scalia Catenacci ${ }^{1}$, Giuseppe Sofi ${ }^{1}$, \\ Ernesto Leva ${ }^{2}$ \\ ${ }^{1}$ Anesthesia and Pediatric Intensive Care Unit and ${ }^{2}$ Department of Pediatric Surgery, Fondazione IRCCS Ca' Granda - Ospedale \\ Maggiore Policlinico, Milan, Italy.
}

\section{Corresponding Author:}

Dr. Stefania Franzini

Email: stefania.franzini@tiscali.it

This is an Open Access article distributed under the terms of the Creative Commons Attribution License (creativecommons.org/ licenses/by/3.0).

Received : February 11, 2019

Accepted : May 8,2019

Published : May 15, 2019

\begin{abstract}
Background: Complications associated with central vein cannulation (CVC) in the adult and pediatric population are well known. On the contrary, adverse events following peripheral vein cannulation (PVC) are rare. Case Report: A 13 months infant was admitted to the Emergency Department for bowel obstruction and severe dehydration. A right external jugular vein access was positioned and fluid infusions started. After few hours the infant became tachypnoic, tachycardic with high lactate levels. An X-ray of the abdomen showed air-fluid levels. The infant was successfully resuscitated following cardiac arrest. A lung ultrasound and a chest X-ray showed a massive right pleural effusion. A chest tube was placed in the right pleural cavity and clear fluid under pressure was drained, which was almost the amount of fluid infused through the right PVC, with stabilization of the general condition. Conclusion: Cannulation of the external jugular vein in infants may result in severe respiratory and cardiac complications and always require adequate monitoring of the patient.
\end{abstract}

Keywords: Central Venous Catheterization, Heart Arrest, Hydrothorax, Jugular Veins, Peripheral Catheterization, Pleural Effusion.

\section{Introduction}

Complications associated with central vein cannulation (CVC) in the adult and pediatric population are well known, the commonest being pneumothorax, hydrothorax, cardiac tamponade, mediastinal perforation, Bernard-Horner syndrome, infection, and hematoma [1-6]. On the contrary, adverse events following peripheral vein cannulation (PVC) are rare [7-11]. We describe a case of an infant who developed a massive hydrothorax following cannulation of the right external jugular vein.

\section{Case Report}

A 13-month-old female infant $(6.7 \mathrm{~kg})$ was transferred to our Emergency Department (ED) from another hospital due to bowel obstruction and severe dehydration. She had a history of severe intrauterine growth restriction and chromosomal abnormalities including short arm deletion of chromosome 1 (region 1 p36.33-p36.23) and chromosome 20 duplication (region 20q 13.33). The patient was delivered preterm at 34 weeks by cesarean section and presented with low birth weight $(1.6 \mathrm{~kg})$, clinical features of severe encephalopathy, psychomotor retardation, epilepsy, blindness, deafness and hypothyroidism, and was admitted to the Neonatal Intensive Care Unit immediately after birth. During admission, she developed bowel obstruction and aspiration pneumonia. At laparotomy small bowel volvulus secondary to malrotation was diagnosed and derotation was performed. At three months of age, 
the patient was discharged home on valproate, phenobarbitone, levothyroxine, ranitidine and vitamin D. In addition, she entered a nutritional program and follow-up.

The day before admission the patient had a single episode of diarrhea and several episodes of vomiting, but no fever. Blood samples and blood gas analysis taken at the referring hospital showed metabolic acidosis (lactate: $3.6 \mathrm{mg} / \mathrm{dL}$, base excess: $4.2 \mathrm{mmol} / \mathrm{L}$ ). On admission to our ED she was lethargic, hypotonic, tachycardiac and severely dehydrated. On examination, the chest and abdomen were unremarkable. A nasogastric tube and a Foley catheter were threaded, while no peripheral venous access could be obtained. The patient was therefore moved to the pediatric intensive care unit (PICU) in order to get a venous line. The attending physician eventually positioned a $22 \mathrm{G}$ peripheral venous catheter PVC in the right external jugular vein and started an infusion of $5 \%$ glucose with $\mathrm{NaCl}(100 \mathrm{mEq} / \mathrm{L})$, at a rate of $20 \mathrm{~mL} / \mathrm{h}$. The patient was subsequently transferred to the pediatric ward, where during the night, had additional episodes of vomiting. A X-ray of the abdomen showed multiple air-fluid levels in the small bowel. Fasting was confirmed and the infusion rate increased to replace fluid losses. Few hours later she became dyspneic, tachypneic, tachycardiac and hypoxic $\left(\mathrm{SpO}_{2}: 92 \%\right)$. The abdomen became distended and tender, and the surgical consultant decided to perform a laparotomy. Back to PICU, on the way to operation theater, she presented a Glasgow Coma scale of 14, respiratory distress, hypoxia $\left(\mathrm{SpO}_{2}: 93 \%\right)$, tachycardia but normal blood pressure and capillary refill time. The intra-abdominal pressure (IAP) was $7 \mathrm{mmHg}$ and normal urine output was maintained. Because of the fast clinical deterioration, the intensivist proceeded with intubation. During induction, the patient experienced severe hypoxia followed by cardiac arrest. Cardiopulmonary resuscitation was promptly performed and return of spontaneous circulation (ROSC) with sinus rhythm was attained in 2 minutes. Under ultrasound (US) guidance, a central venous catheter ( $4.5 \mathrm{Fr} ; 8 \mathrm{~cm}$; double lumen) was positioned in the left internal jugular vein. Following the maneuver, a lung US and a chest X-ray confirmed the accurate position of the CVC, but also showed a massive right pleural effusion [Fig.1]. A chest tube promptly placed in the right pleural cavity swiftly drained $300 \mathrm{~mL}$ of clear fluid under pressure, which was almost the amount of fluid infused through the right PVC. A gas analysis of the effluent was consistent with a mix of glucose and saline solutions ( $\mathrm{pH} 7.25$, glucose $>750 \mathrm{mg} /$ $\mathrm{dL}, \mathrm{Na} 117 \mathrm{mEq} / \mathrm{dL}$ ). Following the uneventful removal of the right $\mathrm{PVC}$, the patient reached the OT, where she underwent laparotomy and division of adhesions. On the third post-operative day the chest tube was removed and the patient extubated, requiring supportive non-invasive ventilation for two more days. The patient resumed oral feeding on the sixth post-operative day and the following day moved to the pediatric ward in good general condition.

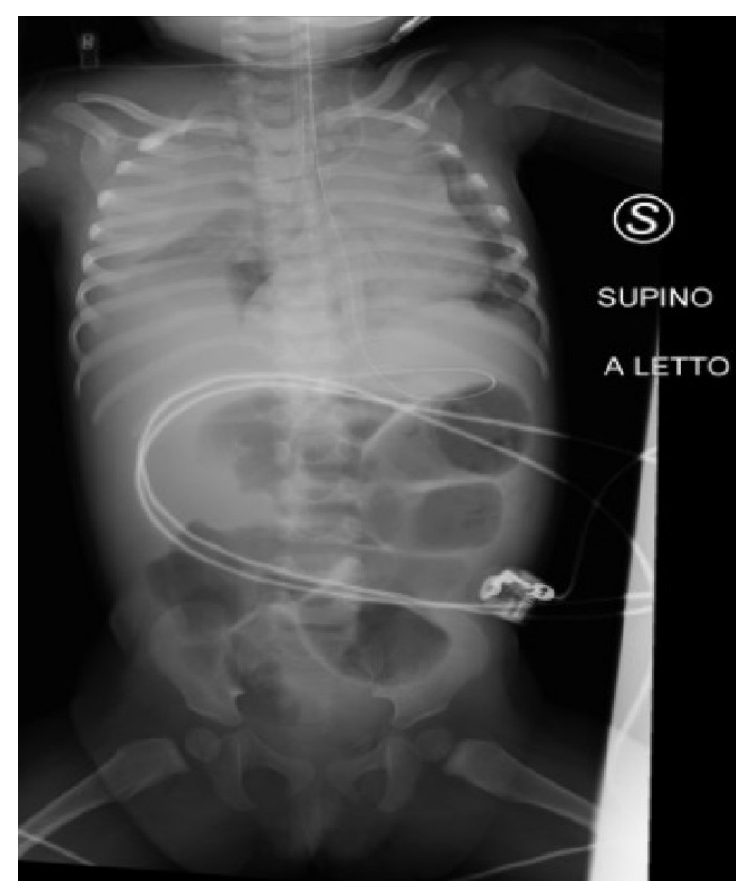

Fig.1: Chest X-ray showing right pleural effusion. 


\section{Discussion}

Cannulation of the external jugular vein is deemed extremely safer than that of the internal jugular vein, because of its superficial location and of the distance from vital anatomical structures (trachea, pleura, lungs and major vessels) [8-10]. Pneumothorax, hydrothorax, Bernard-Horner Syndrome, infection, hematoma, diaphragmatic palsy, and pericardial effusion with cardiac tamponade are potential complications of CVC positioning [1-6]. However, they are extremely rare following PVC, only few cases having been reported in literature [7-11]. Bitar [8] described a case of massive bilateral hydrothorax in a newborn, who developed respiratory distress several hours after a superficial jugular vein cannulation. Kumar [9] reported a case of unilateral iatrogenic hydrothorax, with mediastinal shift, after left superficial jugular vein catheterization in a newborn. In this case, the diagnosis of hydrothorax followed the occurrence of severe respiratory distress, and both the amount and the biochemical composition of the effusion were consistent with the fluids infused through the PVC. Lee [10] reported a case of hydro-mediastinum and cardiac tamponade due to dislocation of a CVC inserted through the right internal jugular vein.

The incident described in this report is the first documented case of cardiac arrest secondary to hydrothorax following cannulation of an external jugular vein in an infant. The hydrothorax may have resulted from direct pleural puncture or from catheter displacement, secondary to manipulation or patient's movements [8]. On turn, the cardiac arrest during induction of anesthesia may have followed the severe hypoxia from lung atelectasis or the extrinsic ventricular compression caused by the hydrothorax. Proper clinical chest examination, earlier chest X-Ray check, lung US, or an adequate hemodynamic and respiratory monitoring $\left(\mathrm{SpO}_{2}\right.$, $\mathrm{ECG}$ ) in the ward might have detected the hydrothorax in advance, allowing to stop fluid administration as soon as the respiratory distress appeared.

\section{Conclusion}

Cannulation of the external jugular vein in infants may result in severe respiratory and cardiac complications and always require adequate monitoring of the patient [12].

Contributors: SF, CC, SS designed and conducted the study. $\mathrm{SF}, \mathrm{CC}$ and EP collected the data and wrote the manuscript. SF, EL, critically revised for important intellectual contents. SF will act as guarantor of study. All authors have read and approved the final manuscript.

Funding: None; Competing interests: None stated.

\section{References}

1. Lin Wang, Zhang-Suo Liu, Chang-An Wang. Malposition of central venous catheter: presentation and management. Chin Med J. 2016;129:227.

2. Hasija N, Hazarika AJ, Goyal K. Massive hydrothorax with malpositioned central venous catheter - Ultrasound detection. Egypt J Anesth. 2016;32:229-231

3. Galofre J, Agusti C, Montserrat JM. Horner syndrome originating from catheterization of the internal jugular vein. Rev Clin Esp. 1988;182:396-397.

4. Hoffmann W, Weidmann B, Jansen W, Tauchert M. Infusion hydrothorax as a sequel of malposition of an internal jugular vein catheter. Med Klin. 1988;83:496498.

5. Van Haeften TW, van Pampus EC, Boot H, Strack van Schijndel RJ, Thijs LG. Cardiac tamponade from misplaced central venous line in pericardiophrenic vein. Arch Intern Med. 1988;148:1649-1650.

6. Meoni MS, Paoletti F. A complication of central venous catheterization: penetration into the mediastinum. Minerva Anestesiol. 1988;54:63-66.

7. Finley GA. A complication of external jugular vein catheterization in children. Can J Anaesth. 1988; 35:536537.

8. Bitar FF, Obeid M, Dabbous I, Hayek P, Akel S, Mroueh S. Acute respiratory distress associated with external jugular vein catheterization in the newborn. Pediatr Pulmonol. 2003;36:549-550.

9. Jangid MK, Yadav SK. Rare case of massive hydrothorax following external jugular vein cannulation. Pediatric Oncall Journal. 2014;11.

10. Lee TS, Chen BJ. Hydromediastinum following insertion of a central venous pressure line via the right external jugular vein. J Clin Anesth. 1993;5:436-438

11. Gajre G, Ali SD. Mediastinal tamponade following external jugular vein cannulation. Indian Pediatr. 2001;38:1177-1180.

12. Pettit J. External jugular cannulation in infants and children. J Infusion Nursing. 2009;32:93-97. 